\title{
The Practical Inquiry of the AC Withstand Voltage Test Technology of the Same Frequency and Phase Based on the GIS Equipment
}

\author{
Yuan Ting, Xu Bingfu, Gu Qiang, Chen Bibo \\ Panjin Power Supply Company, Liaoning Electric Power Company Limited, State Grid, China,
} 16295036@qq.com

\begin{abstract}
Keywords: GIS equipment, the withstand voltage test of alternating current with the same frequency and phase, practical application.
\end{abstract}

\begin{abstract}
This paper analyzed the basic principles of the alternating current withstand voltage test technology work of the same frequency and phase.And at the same time analyzed the danger that exist during the process of the test that basic on the GIS equipment,put forward corresponding preventive and control measures.Analyzed the applicability of the ac withstand voltage test of the same frequency and phase technology by taking the single busbar connection mode and double busbar connection mode of GIS equipment for example meanwhile,so that the GIS equipment can get promotion and development.
\end{abstract}

The GIS equipment has the advantages of cover a small area, simple operations, therefore it is widely used in the power grid construction in China.However many problems have emerged in the process of promoting GIS equipment,part of the problems even greatly influenced the power supply reliability of the power grid.Nowdays, our country power supply load increased gradually, grid also need optimization, a large number of GIS equipment have started to be reconstruction or expansion.In the extension and reconstruction work,construction people must stop the GIS bus working, which would cause certain influence to the power supply.Therefore, it is urgently needed to carry out ac withstand voltage test of the same frequency and phase technology, in order to avoid the phenomenon that all the GIS bus stop running during the process of GIS equipment reconstruction or expansion.

\section{The basic principles of the alternating current withstand voltage test technology of the same frequency and phase}

The equipment of the ac withstand voltage test is a new designed pressure device, which improved and perfected from the original feeling series resonance device and supported by the principle of the same frequency and phase theory. The reference signal of the ac withstand voltage test of the same frequency and phase is the the voltage signal generated by the bus of the operating state PT.Using linear push-pull amplification and phase-locked loop technology to make the device generating the test voltage, which has the same frequency and phase with the operation state busbar voltage.

In order to obtain phase reference signal,the AC voltage test equipment of the same frequency and phase would filter and match the single from the PLL.Then put the phase reference signal to the phase comparator and adjust the phase reference signal until the sampling signal and the second signal of PT has the same frequency and the phase difference value between them within the allowable so that the entire device form a locked state based on the same frequency, phase and voltage. Moreover,the protection monitoring of the same frequency and phase AC voltage test device could test the test voltage applied to the GIS fracture. If the test voltage was more than the protection setting value set by the user or the detected phase difference value was too big, the device would notify the user through the fault signal so that the user to discover timely.Meanwhile, the same frequency and phase AC voltage test equipment is able to disconnect the power inverter output channels promptly,to stop putting pressure on the sample. 
When the same frequency and phase AC voltage test equipment is in the same frequency and phase state, the voltage waveform generated for isolating the switch fracture is shown in Figure 1.Suppose the voltage generated by the operation process as U2, and the voltage produced during the test as U1.Then the absolute value of the difference between the two is not only the voltage that carried by the running part, but also the voltage carried by the isolation switch at the connected position of the space.Compared to the voltage generated from the space of the isolation switch,the value is smaller.So there wouldn't be breakdown at the fracture position of the isolation switch.And it is impossible Can to cause an obstruction to other running GIS equipment.

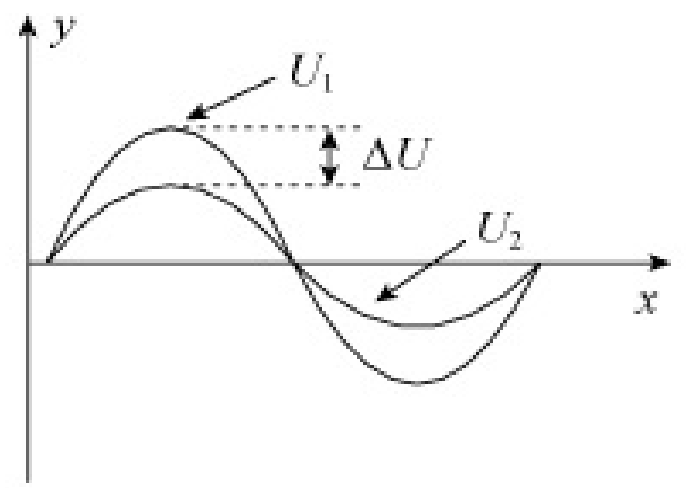

Figure 1. schematic of the voltage waveform at the fracture position of the isolation switch(the same frequency and phase)

\section{Dangerous points and preventive measures of the GIS devices during the same frequency and phase AC voltage test}

Normally, when the laboratory personnel perform the same frequency and phase AC voltage test process,the withstand voltage test part and the original run part are separated only by the use of isolation switch.Therefore,Designers need to analyze the danger point exists during the test of the same frequency and phase AC voltage, and propose appropriate preventive and control measures to ensure that laboratory personnel, GIS equipment and the power grid would not be threatened.

\section{PT secondary signal sampling issues}

During laboratory personnel performing the PT secondary signal sampling process,it is prone to find signal phase wrong, short or non-dot end and so on.Therefore, laboratory personnel should follow the following steps for prevention and control of these problems.First of all, the the experimenter should do measurement work of the both sides' resistance and the signal line insulation resistance of the secondary signal simulation unit every time before the same frequency and phase AC voltage test to ensure the normal operation of PT signal. Secondly, the power supply bureau should appoint professionals to the scene to detect and guarantee the PT secondary signal, only after the PT secondary signal be confirmed by both, it could be used.During the work, the experimenter should appoint persons specialized in protection devices to avoid other people touching.

\section{Bus ground fault}

Bus earth fault is usually generated by the emergence of a running bus side switch chamber insulation breakdown phenomenon. If it is bus differential protection or line spacing switch refuses to start, it will make a power leapfrog tripping , and cause large accidents easily. For this reason, construction workers can indicate the length of time between the running down line protection side and the II segment on the pressure test maintenance outage way documents.Relay protection personnel shall test the bus differential protection to ensure it is normal operation, and then do the withstand voltage test. 


\section{The applicability of the same frequency and phase AC withstand voltage test}

\section{GIS space expansion and overhauling under the single busbar connection mode}

According to these equipments, after one or several intervals after expansion, rebuilding, or overhauling, staff can start the same frequency and phase AC voltage test. Staff turn the test voltage through the expansion or maintenance of the interval outlet casing,and ensure the stable operation of the bus at the same time. At this time, the staff can turn off the bus side to isolate the switch DS2, the bus voltage transformer secondary terminals etc which are in the running state to obtain reference voltage.

\section{GIS space expansion and overhauling under double bus connection mode}

For double bus connection mode GIS equipment, when one or more intervals have been expanded and inspected, staff will be able to do the same frequency and phase ac withstand voltage test. The staff turn on the test voltage through the expansion of the interval or overhaul interval on the wire.Among them, the test sleeve can be mounted on the spacer of the cable outlet ,or the staff also could access voltage through other qualifying interval. Ensure that the original bus I , bus II runs in a stable condition.At this time, the staff would turn off the switches DS2 and DS3 on the bus side which are used for partition.Moreover, the staff needs to disconnect the shunt between the bus coupler and the switch used to isolated. And at the same time, obtains the reference voltage from the running section of bus I, bus II and so on.

\section{GIS bus overhauling under Double bus connection mode}

For GIS equipment under this mode, when the bus finish overhauling, the same frequency and phase ac withstand voltage test should be done like this:test voltage access the device through the line spacing, and then cut off the fault bus bar, power of bus I,and then convert all load to bus II as summary, turn off the isolation switch DS2 at the same time, and close DS2. In this case, the mother associated interval and the isolation switch have been closed, some staff obtains the reference voltage from the running section of II female voltage transformer secondary terminals, etc.

\section{GIS bus and the interval expansion under Double busbar connection mode}

For this kind of equipment, the work od this type of GIS interval and the expansion of the bus bar of the same frequency and phase ac withstand voltage test should be divided into two parts.The first part of work is as follows: first of all, the staff should make bus I to carry the load of total station, then turn off the power supply of bus II, connect it to the expansion device of bus II . After connection, close the isolation switch DS2 on the II female side and the expansion bay circuit breaker, turn off the isolation switch DS3 on the I female side. And at the same time, turn off the bus coupler bay circuit breaker and the isolation switch DS4/DS5. The test voltage was turned to the equipment through the expansion or maintenance of the interval outlet casing to start the same frequency and phase AC voltage test of expansion interval and II female extension. Then staff could obtain the reference voltage from the running section of I female voltage transformer secondary terminals, etc. The second part of the work is substantially the same with the first part of the work , just expand female II according to the first part of the work program.

\section{Conclusion}

Compared with the traditional GIS equipment modification, the same frequency and phase ac withstand voltage test technology applied in GIS equipment would make the GIS equipment more secure in the process of expansion and rebuilding. It would also be applied to various types of GIS equipment, the practical value is higher.And play an active role in the power grid construction and development of our country.

\section{References}

[1] Wu wei,application study of GIS equipment of the same frequency and phase ac withstand voltage test technology J.Science innovation and application, 2014, 4:164-165. 
[2] Yin Hua,Wang Qian,Long Yingkai.Questions in GIS equipment of the same frequency and phase ac withstand voltage test technology J.Smart grid, 2015,07:679-682.

[3] Wu Gaolin,Xu Ruilin,Xiao Qianbo.Simulation analysis and prevention measures of the bus bar insulation breakdown in the AC withstand voltage test of GIS equipment J.High voltage apparatus, 2013,04:98-104.

[4] Sun Liang.Ningxia power grid for the first time to carry out the same frequency and phase AC voltage test J.Ningxia electric power, 2015,04:73.

[5] Li Zhongsheng. Calculation of the set of the reactor in series resonant voltage withstand test J. Journal of Hunan Industry Polytechnic, 2014,01:4-6. 